\title{
The use of models to explore IPM strategies and design pest suppressive landscapes for sustainable agricultural practice
}

\author{
$\underline{\text { Hazel R. Parry }}$, Nancy A. Schellhorn ${ }^{a}$ \\ ${ }^{a}$ CSIRO Ecosystem Sciences, Brisbane, QLD, Australia \\ Email: hazel.parry@csiro.au
}

\begin{abstract}
In order for agriculture to move from an unsustainable reliance on broad-spectrum chemical pesticides to a more sustainable future, we need make a transition to a 'total system' approach. Agricultural pest problems need to be considered beyond the crop boundary, and we need to understand the role of the landscape matrix for the suppression of pests in crops as well as providing other ecosystem services, such as pollination.
\end{abstract}

We combine field-based survey data with a spatially-explicit simulation model to assess the benefits of integrated pest management strategies at the landscape scale. We explore the features of pest suppressive landscapes for a native insect pest in Australia, the Rutherglen Bug (Nysius vinitor). The model is designed to explore spatially the effects of environmental drivers including temperature, habitat type and habitat quality, on the population dynamics and dispersal of the bug. This drives its landscape pest status for a given season and location.

We present scenarios evaluating pest control benefits that are likely to be achieved from managing native remnants by weed removal at multiple spatial scales. Our results indicate that the spatial location of weedy pasture in relation to the crop appears to be highly important in determining the density of $N$. vinitor within the crop (outweighing the effect of the overall proportion of weedy non-crop habitat in the landscape), and this warrants further exploration.

Our approach as illustrated in this paper, using models combined with field data to explore Integrated Pest Management (IPM) strategies and design pest suppressive landscapes, will allow farmers to optimize multiple ecosystem service benefits by i) understanding both the hazards and benefits of non-crop vegetation in the landscape ii) providing a tool to help plan the extent and location of re-vegetation plantings. This work has potential to influence agricultural land use policy in Australia, with further work planned to model the implications of landscape change and non-crop habitat management strategies for multiple ecosystem services.

Keywords: Integrated Pest Management, Landscape Ecology, Sustainable agriculture, Cohort-based, Spatially-explicit, Ecosystem services, Dispersal 
Parry H.R. et al., The use of models to explore IPM strategies and design pest suppressive landscapes for sustainable agricultural practice

\section{INTRODUCTION}

\subsection{The ecosystem service of natural pest control}

Natural pest control is an important ecosystem service that directly benefits agriculture (Losey and Vaughan, 2006). Mobile pests do not recognise field or farm boundaries, and therefore attempts at controlling them using field-based approaches have often failed. In addition, some landscapes appear less prone to invertebrate pest infestations than others, suggesting that there are features of landscapes that may be managed to create more 'pest suppressive landscapes'. Understanding the appropriate spatial scale for controlling pests and creating a pest suppressive landscape requires knowledge of the ecological function of the habitats present in the landscape and a basic understanding of how pest and natural enemies move in agricultural landscapes. To address this, studies are required that acknowledge that agricultural pest problems need to be considered beyond the crop boundary (Schellhorn et al., 2008) and that the landscape matrix (i.e. non-crop habitat) matters for the suppression of pests in crops as well as providing other ecosystem services, such as pollination (Bianchi et al., 2006).

Field-based approaches can play an important role in understanding where and when pests and beneficial arthropods are present in our landscapes, and how they move between habitats. However, these can generally only be undertaken at limited spatial scales and over limited time frames. Laboratory studies can also provide complimentary data, such as detailed observations on a species' life history and to explore important traits, such as environmental drivers of dispersal behaviour. By integrating field observations and data from laboratory studies with spatially-explicit ecological models, we can overcome several of the limitations of a purely empirical approach to studying ecosystem services in agricultural landscapes: models allow us to explore and generate hypotheses at multiple landscape scales, both integrating data and also communicating the synthesis of the data with the model, giving often profound theoretical insights (Parry et al., 2012).

This paper takes the case study of a particular insect pest, the Rutherglen Bug (Nysius vinitor), and demonstrates the value of combining field observations and laboratory empirical data with a spatially-explicit population dynamics simulation model, in order to explore integrated pest management (IPM) strategies for managing $N$. vinitor outbreaks in agricultural landscapes of Australia. Individual and ecosystem impacts of pest and beneficial populations are difficult to observe and measure in the field. We show how computer simulations allow us to explore the outcomes of potential interventions, such as removal of known pest habitat, and help with operational choices; for example how much and where to implement IPM strategies to have greatest impact.

\subsection{Rutherglen Bug (Nysius vinitor (Hempitera: Orsillidae))}

Although $N$. vinitor outbreaks are known to cause high levels of damage in a wide variety of crops, relatively little is known about the spatial population dynamics of this sporadic native insect pest in Australia. Our national field-based research has helped identify some important information about $N$. vinitor. In particular, in the grain growing regions that we have studied we have identified that the non-crop habitat (native vegetation remnants and pasture) host the bug throughout the year. However, within non-crop habitats $N$. vinitor was found much more commonly on weed plant species than on native plants or grasses - indicating that weed removal in non-crop habitat may be a key IPM strategy to suppress $N$. vinitor populations.

We have combined information we gathered from the field and from past laboratory studies with our newly developed model, to address the following three research questions:

1) What are the features of pest suppressive landscape and their impact on $N$. vinitor pest status?

2) What is the potential effectiveness of field, farm and landscape IPM options in $N$. vinitor suppression? This includes farm landscape configuration and the proportion of non-crop habitat and condition (i.e. weed management) of non-crop habitat.

3) At what spatial scale would landscape management prove most effective at reducing $N$. vinitor pest outbreaks?

\section{FIELD-BASED RESEARCH}

The model presented in this paper was validated using outputs from a large two-year field study in three states of Australia: New South Wales (NSW), Queensland (QLD) and Western Australia (WA). Monthly pest and beneficial arthropod species density data was collected from two agricultural landscapes in each region 
Parry H.R. et al., The use of models to explore IPM strategies and design pest suppressive landscapes for sustainable agricultural practice

from multiple crop and non-crop habitats, replicated both within and between landscapes. Analysis of the field data identified important habitat and plant host information on $N$. vinitor as described above, that has been used to initiate the model $(N$. vinitor density and location). The NSW and QLD field data is used to validate patterns produced by the model: both by comparison with the model predicted temporal density of $N$. vinitor over the two year study period (Figure 3) and also by comparison with the model predicted difference in the density of $N$. vinitor in Sorghum between the QLD landscapes (Figure 6).

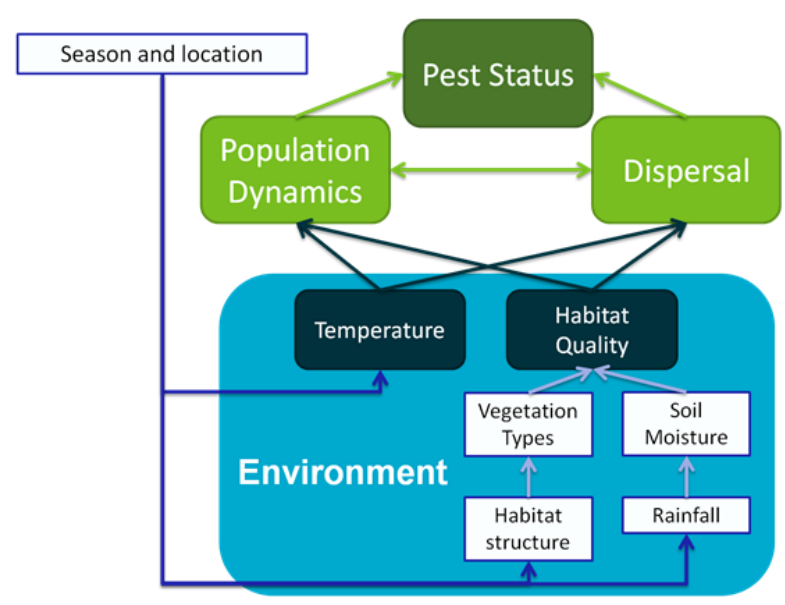

Figure 1: Conceptual diagram of the model

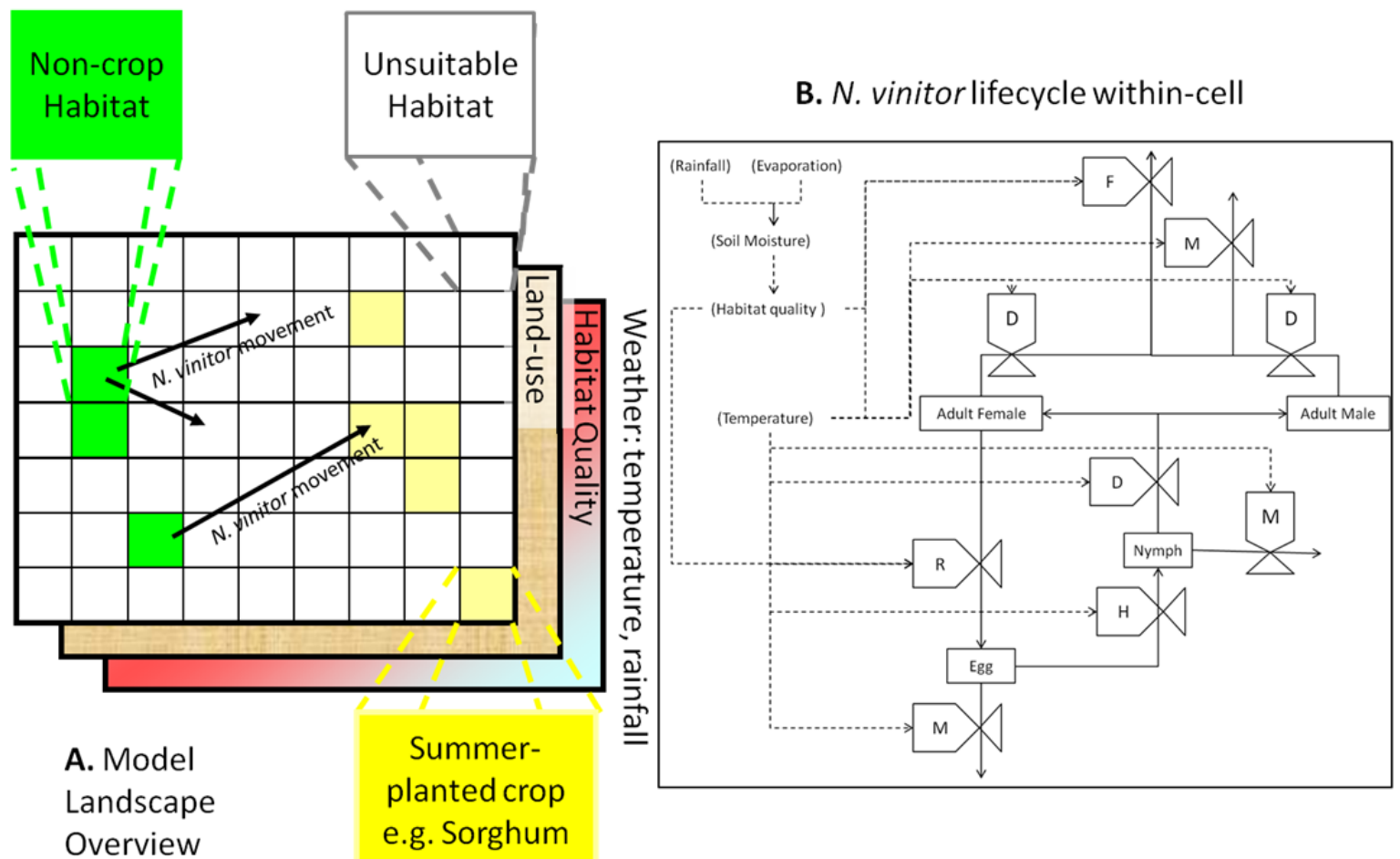

Figure 2: A: Overview of the $N$. vinitor model landscape. The spatial-temporal input layers of key environmental drivers, including habitat types (land use), habitat quality and weather (temperature, rainfall and evaporation), drive the simulation of cohort population dynamics within the cell (see B), and also cellcell movement. B: Relational diagram of the $N$. vinitor lifecycle model. Rectangles denote state variables,

brackets driving variables and valve symbols factors affecting rates of flow. Solid arrows are a flow of energy or matter; dashed arrows denote an effect of one factor on another. H, egg development rate/hatching rate; $\mathrm{D}$, development rate (differs for nymphs and adults, and between male and female adults); $\mathrm{R}$, reproduction rate/rate of egg laying; F, flight; $\mathrm{M}$, mortality rate.

\section{MODEL OVERVIEW}

There are no existing lifecycle models of $N$. vinitor population dynamics. Using a combination of the available literature and our own field data for validation, a novel cohort-based model has been developed and extended as a spatially-explicit representation of the population dynamics and dispersal of $N$. vinitor in agricultural landscapes of Australia. This allows us to explore large scale, regional differences between population dynamics and understand the drivers of such differences as well as to explore detailed local impacts of landscape configuration that can lead to outbreaks of the bug. The model incorporates the effects 
Parry H.R. et al., The use of models to explore IPM strategies and design pest suppressive landscapes for sustainable agricultural practice

of environmental drivers including temperature, habitat type/land use and habitat quality, on the population dynamics and dispersal of the bug, leading to its pest status for a given season and location (figure 1).

The model has been developed using the Java programming language. The model treats the landscape as a grid (where any habitat data can be read into the model as an ASCII file). The model is initialised with a 'background' population of adult $N$. vinitor at a density of $0.1 \mathrm{per} \mathrm{m}^{2}$, scaled to the cell size. If the cell is suitable habitat then the cell population is not allowed to drop below this 'background' level. The model iterates through the grid and simulates population dynamics in each cell of the grid using a cohort-based approach, adjusted to the cell size. The model runs on a daily time step. At each daily timestep cohorts are updated. During the timestep, mortality and development occurs at each of three lifestages (egg, nymph and adult) and reproduction takes place. Adult flight is initiated and flight distances calculated according to several environmental drivers (figure 2). Adult cohorts that take flight are then moved to a temporary cohort at their destination cell, which is iterated and moved to the main cohort list at the next model iteration. Apart from the flight calculations, which are drawn from a random distribution (see section 2.2), the model is deterministic, based on equations derived from empirical relationships to driving variables.

\subsection{Flight simulation}

$N$. vinitor adults fly during both the day and the night (Kehat and Wyndham, 1973a). The peak flight activity is observed to be late evening. Flight activity is thought to be constant and proportional to the population size, however mass flights occur when certain weather conditions exist (Kehat and Wyndham, 1973a) - these have been observed to relate to fluctuations in temperature around a threshold and drought. Both males and females disperse, however it is believed that there are differences in movement patterns between immature and mature females as well as between the sexes. Nymphal movement is negligible (Ramesh, 1984).

A threshold temperature of $19^{\circ} \mathrm{C}$ is assumed, below which flight will not occur (Kehat and Wyndham, 1973a). The quality of food availability is assumed to vary with soil moisture, as flight is known to relate to food and water availability (Kehat and Wyndham, 1973a). Therefore in the model, take-off is assumed to be inversely proportional to soil moisture (where soil moisture ranges from $0(\min )-1(\max )$, based on a simple 1-layer soil moisture model dependent on rainfall and evaporation).

To allow for effects beyond the simulation landscape we simulate the landscape as a torus, therefore a long distance flight departing the landscape is randomly returned to a cell within the landscape (essentially representing a long-distance migrant initiated elsewhere). Given that flight occurs, using estimates for flight time under conditions of no food and water, assuming a maximum flight speed of $3 \mathrm{~km} / \mathrm{hr}$, the cumulative percentage of adult flight times is used to derive an exponential flight distance curve, which allows for a random number to be selected to estimate flight distance $\left(\mathrm{D}_{\text {flight }}\right)$ in metres (based on data from Kehat and Wyndham, 1973b):

$$
D_{\text {flight }}=59 e^{3 x}
$$

Where $\mathrm{x}$ is a random number between 0 and 1 , essentially the probability of reaching a particular distance (with a maximum distance $(\mathrm{x}=1)$ of $1179 \mathrm{~m}$ and minimum $(\mathrm{x}=0)$ of $59 \mathrm{~m})$. Flight direction is assumed to be random, although wind effects are known to occur (Kehat and Wyndham, 1973a). Timing of crop sowing is
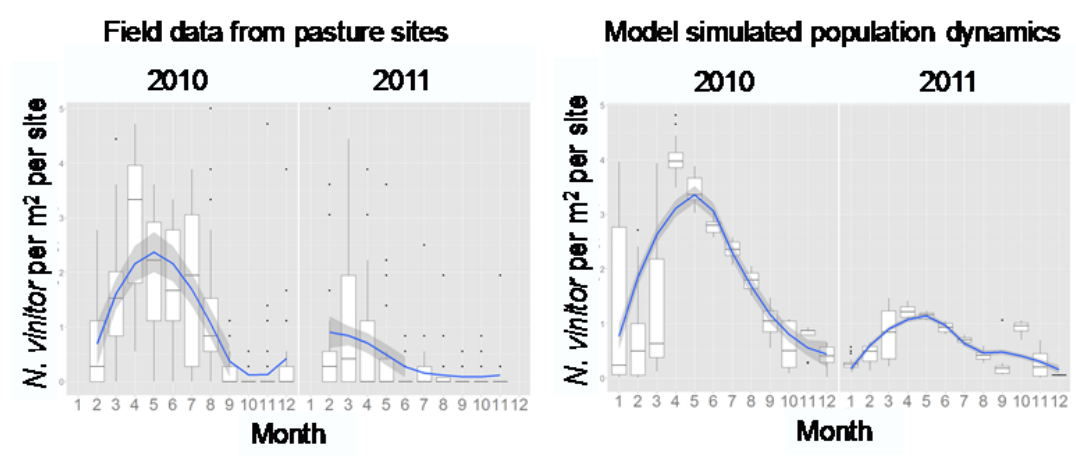

Figure 3: Adult $N$. vinitor observd field density (left) compared to model simulated density (right), pasture NSW 2010-2011. Error bars show the standard error around the monthly mean for both the field data and the model. thought to have no effect on flight activity timing (Ramesh, 1984), therefore the drivers are assumed to be purely climatic.

Movement is
implemented in the
model for each cohort
by firstly determining
whether the cohort has
already dispersed
(assuming dispersal
occurs only once for a
cohort). If the
maximum daily


Parry H.R. et al., The use of models to explore IPM strategies and design pest suppressive landscapes for sustainable agricultural practice

temperature is above the threshold of $19^{\circ} \mathrm{C}$, a random probability of movement is calculated based on 1-soil moisture (i.e. a greater propensity to move when soil moisture is low). The entire cohort is then moved to another cell. This is in preference to splitting and rejoining cohorts to simulate movement, as it was found this introduced large errors in population density due to increasingly large numbers of cohorts with small cohort sizes, and the computational need to remove cohorts from the model when they become too small. If cohorts were merged on arrival in a new cell (e.g. by taking the mean parameters of several cohorts) this introduced additional problems, including overestimation of lifespan/development time. As populations of $N$. vinitor in these landscapes are sufficiently large, with a large number of cohorts per cell, the approach of moving whole cohorts is considered to pose less risk of artificial 'clustering' (cf. issues with spatial 'Super-individuals' in individual-based modelling (Parry and Evans, 2008)).

\section{RESULTS}

The within-cell dynamics of the model were validated against field data from 2010/2011. We found the model fitted closely with data from the field, providing simulated mean densities and population growth estimates within the (highly variable) range observed as shown (figure 3), but also in terms of the magnitude/timing of flight (not shown).

The model was run on two contrasting landscapes situated on the Darling Downs in Queensland, where we had also gathered monthly field data over a two year period (figure 4). A corresponding meteorological data file was downloaded
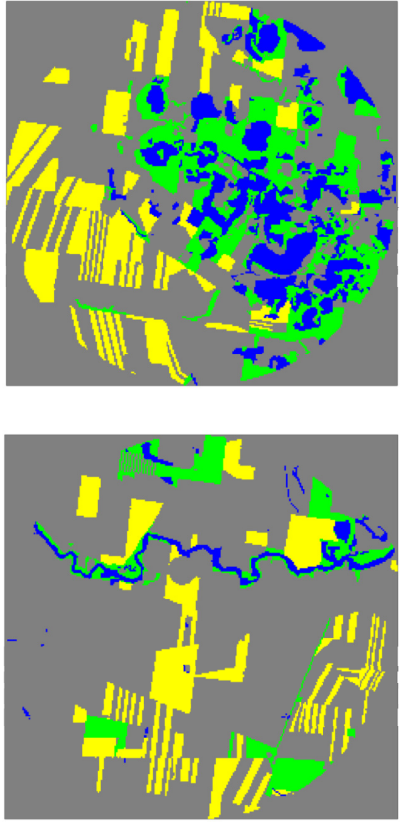

B

Figure 4: Simulation landscapes, $\mathrm{A}=$ Irongate, $\mathrm{B}=$ Bowenville, showing the distribution of the key habitat types:

Sorghum, Pasture and Native Vegetation Remnants (NV). from SILO ${ }^{1}$ to provide weather data inputs from nearby weather station 41082 (Pittsworth).

Despite Irongate having a much higher percentage of source habitat (figure 4), the simulated density of $N$. vinitor in sorghum is very similar in both landscapes (figure 5). This is also what we expect from the field data (figure 6). In fact, we expect the lower vegetation landscape (Bowenville) to have a higher $N$. vinitor density in sorghum as this was observed in the field. The mean density simulated in the sorghı is of a similar magnitude to that found in field data for 2010, although the model predicted lower densitis $B$ an the actual densities found in the field. This lower simulated density would be expected, given that this landscape is not isolated in reality as it is in the model, and a portion of the migrants into sorghum are likely to arrive from elsewhere.
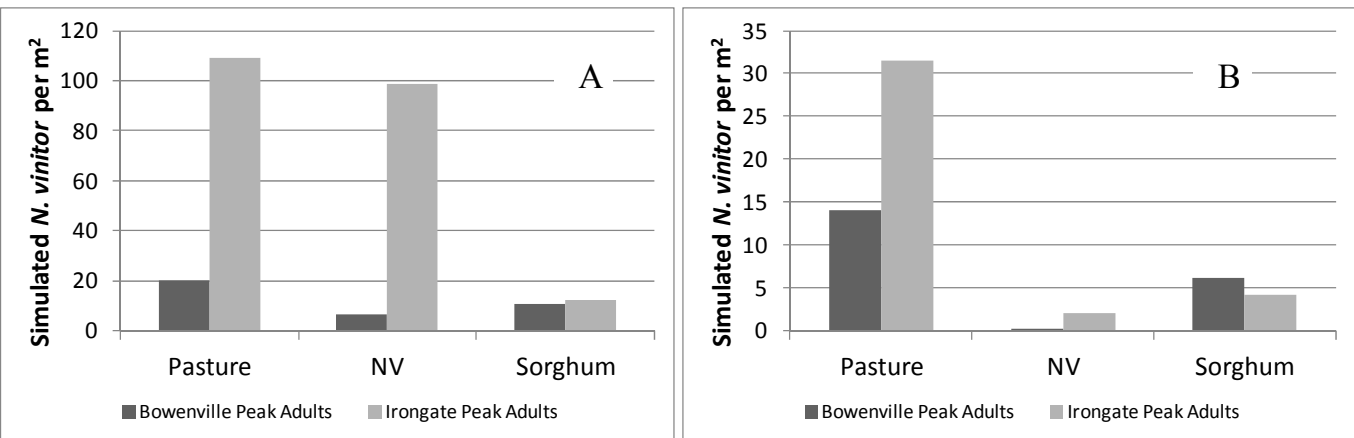

Figure 5: Model simulated N. vinitor mean peak density for each habitat type. A: Simulated densities under condition of weedy non-crop habitat. B: Simulated densities under condition of weeds removed from native vegetation remnants (NV)

\footnotetext{
${ }^{1}$ http://www.longpaddock.qld.gov.au/silo/
} 
Parry H.R. et al., The use of models to explore IPM strategies and design pest suppressive landscapes for sustainable agricultural practice

A

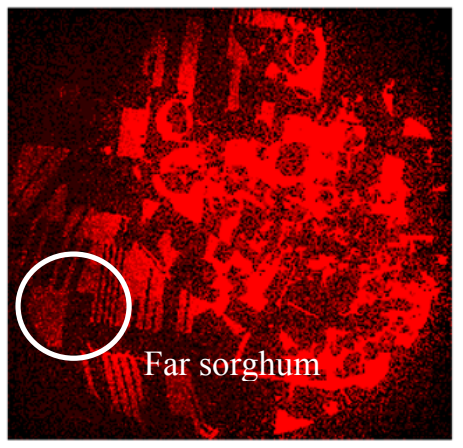

B

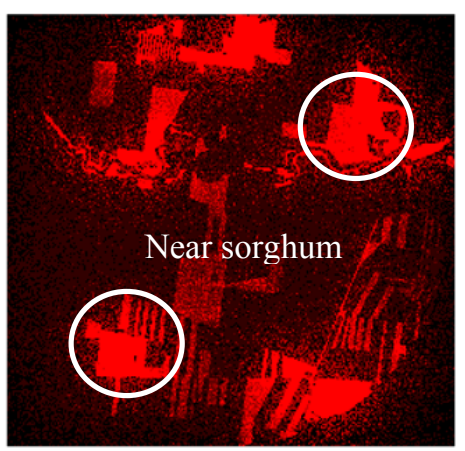

Figure 7: Effect of $N$. vinitor dispersal behaviour in the model, brighter red $=$ higher density of $N$. vinitor, $\mathrm{A}=$ Irongate, $\mathrm{B}=$ Bowenville. Sorghum near to weedy pasture or weedy native vegetation (white circles, B) has higher

$N$. vinitor density than sorghum far from weedy pasture or weedy native vegetation remants (white circle, A) at the landscape scale.

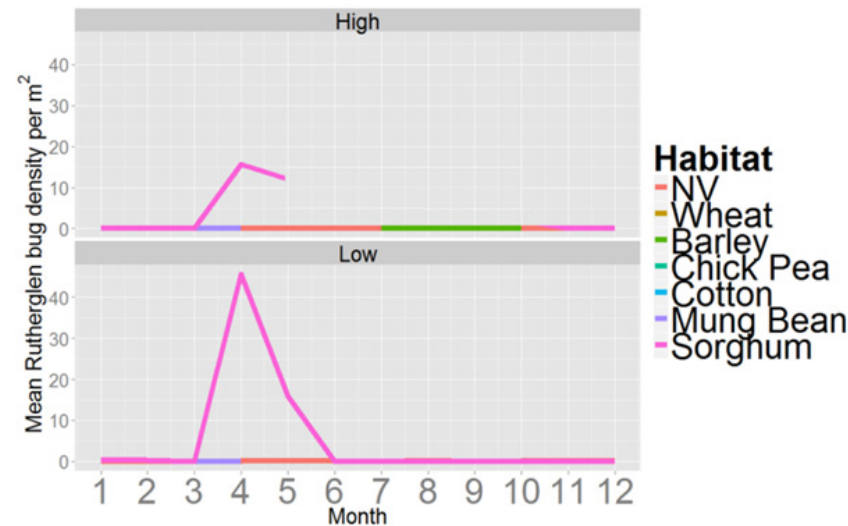

Figure 6: The field density of $N$. vinitor in Sorghum in QLD 2010: the higher density observed in the low native vegetation landscape (Bowenville) is comparable to that simulated by the model (figure 5) as a result of dispersal processes (figure 7).

The effect of distance is even more pronounced when weeds are removed from the native vegetation remnants in the model (figure 5b); we see that although there is more pasture in the Irongate landscape the modelled density in sorghum actually is highest in Bowenville, due to the proximity of $N$. vinitor sources. By examining the spatial model output, the difference between landscapes is evidently due to the effect of distance/connectivity of the crop from the pasture source and the limits to the dispersal capabilities and behaviour of $N$. vinitor as it moves from the source across the model landscape (figure 7). This warrants further exploration, and this will be undertaken with a series of artificial landscapes of varying connectivity and pasture/crop configuration, to quantify the relationship between connectivity to weedy non-crop habitat and crop pest density for $N$. vinitor.

\section{DISCUSSION AND CONCLUSIONS}

The model developed for $N$. vinitor provides a template for further exploration of the implications of landscape configuration for a range of pest and predator species. The results of our model show that $N$. vinitor densities can build in weedy pastures and native vegetation remnants to high densities during summer and autumn, if climatic conditions (and therefore habitat condition) are suitable. Dispersal events are also triggered by these factors, causing $N$. vinitor to disperse across the landscape. However, evidence from this study indicates that their dispersal is likely to be mostly of a limited distance (ignoring wind effects), generally less than $1 \mathrm{~km}$, and so there is a strong distance effect on their dispersal.

Landscapes with a high percentage of weedy non-crop habitats will have a high overall density of $N$. vinitor. However, we find no evidence that this determines the $N$. vinitor density in the crop. The spatial location of weedy pasture in relation to the crop appears to be highly important in determining the density of $N$. vinitor within the crop (outweighing the effect of the proportion of weedy non-crop habitat in the landscape), and this warrants further exploration. In terms of landscape management, removal of weeds in native vegetation remnants or pastures is likely to have an impact on $N$. vinitor populations at multiple spatial scales. Populations of $N$. vinitor are likely to be reduced across all crops in the landscape, with the largest effects realized in crops planted close $(<2 \mathrm{~km}$, approx $)$ from the non-crop vegetation.

Australian agricultural landscapes are now in a period of transition. Recent changes to EU legislation are making ripples in Australian government policy. The Australian government is now funding initiatives such as the Biodiversity fund, buffer zones for pesticide spray and Carbon planting. An important aspect that seems to be lacking in this new government policy is exactly how such initiatives should be implemented (Burns and Lindenmayer, 2012). In particular, the questions of what initiatives should have priority, where projects should be placed, and how much is necessary to achieve desired outcomes, are highly important. 
Parry H.R. et al., The use of models to explore IPM strategies and design pest suppressive landscapes for sustainable agricultural practice

Many agricultural landscapes in Australia are subject to environmental stress and in some places have multiple, competing land use functions in an increasingly peri-urban space (e.g. the Northern Rivers region, NSW). We believe that re-vegetation plantings in agricultural landscapes will be most successful if they are multi-functional, providing several ecosystem services with a single re-vegetation planting. A simulation modelling approach is a powerful method to assess the potential impacts of landscape change, because it allows us to perform landscape analysis and land use change 'experiments' that are not possible on the ground. This approach also allows us to optimize multiple ecosystem service benefits by i) understanding both the hazards and benefits of non-crop vegetation in the landscape ii) providing a tool to help plan the extent and location of re-vegetation plantings.

Importantly, we cannot isolate ecosystem services of biological control from other ecosystem services that can be provided by non-crop habitat (Fielder et al., 2008). A major challenge for this new paradigm of landscape design is to consider how we should best synergize multiple functions of non-crop habitat, such as biodiversity conservation, carbon sequestration and pesticide spray buffering, alongside the benefits of biological control.

\section{ACKNOWLEDGEMENTS}

This project is funded by the Australian Grains Research and Development Corporation. It is a collaboration between CSIRO Ecosystem Sciences, the University of Queensland, DEEDI QLD and DAFWA. Many thanks go to Andrew Hulthen for preparing the GIS data used in the modelling, and to Felix Bianchi for his initial input to this project. We thankfully acknowledge the other members of our project team: Art Diggle, Tony Dore, Mike Grimm, Jamie Hopkinson, Sarina Macfadyen, Svetlana Micic, Mick Neave, Paul Yeoh, and Myron Zalucki. The Repast Simphony java-based modelling toolkit provided code and a GUI that helped manage and visualize the spatial data used by the model http://repast.sourceforge.net/repast_simphony.html including classes that implement Geotools http://geotools.sourceforge.net

\section{REFERENCES}

Bianchi, F.J.J.A., C.J.H. Booij and T. Tscharntke, Sustainable pest regulation in agricultural landscapes: a review on landscape composition, biodiversity and natural pest control. Proceedings of the Royal Society B-Biological Sciences 273(1595):1715-1727, 2006

Burns, E. and D. Lindenmayer, The Biodiversity Fund - another missed opportunity? http://theconversation.edu.au/the-biodiversity-fund-another-missed-opportunity-4889, 2012, accessed $04 / 07 / 2013$

Fielder, A.J., D.A. Landis, and S.D. Wratten, Maximizing ecosystem services from conservation biological control: The role of habitat management. Biological Control 45: 254-271, 2008

Kehat, M. and M. Wyndham, Flight activity and displacement in the Rutherglen bug Nysius vinitor (Hemiptera: Lygaeidae). Australian Journal of Zoology 21(3): 413-426, 1973a

Kehat, M. and M. Wyndham, The relation between food, age and flight in the Rutherglen bug, Nysius vinitor (Hemiptera: Lygaeidae). Australian Journal of Zoology 21(3): 427-434. 1973b

Losey, J.E. \& Vaughan, M. The economic value of ecological services provided by insects. BioScience, 56, 311-323. 2006

Parry, H.R. and Evans, A.J. A comparative analysis of parallel processing and super-individual methods for improving the computational performance of a large individual-based model. Ecological Modelling 214: 141-152. 2008

Parry, H.R., Bianchi, F.J.J.A. and Schellhorn, N.A., The use of models to design pest suppressive landscapes for sustainable agricultural practice. International Environmental Modelling and Software Society (iEMSs) International Congress on Environmental Modelling and Software 'Managing Resources of a Limited Planet', Sixth Biennial Meeting, Leipzig, Germany R. Seppelt, A.A. Voinov, S. Lange, D. Bankamp (Eds.), http://www.iemss.org/sites/iemss2012/proceedings.html, 2012, accessed 04/07/2013

Ramesh, P. The ecology and behavior of Nysius vinitor Berg. (Hemiptera-Lygaeidae) - a key pest of sunflower (Helianthus annuus L.). PhD Thesis, University of Adelaide. 1984

Schellhorn, N.A., S. Macfadyen, F.J.J.A. Bianchi, D.G. Williams and M.P. Zalucki, Managing ecosystem services in broadacre landscapes: what are the appropriate scales? Australian Journal of Experimental Agriculture 48:1549-1559 2008 